\title{
Proteomic Definitions of Mesenchymal Stem Cells
}

\author{
Martin H. Maurer \\ Department of Physiology and Pathophysiology, University of Heidelberg, Im Neuenheimer Feld 326, 69120 Heidelberg, Germany
}

Correspondence should be addressed to Martin H. Maurer, neurogem@googlemail.com

Received 4 August 2010; Accepted 17 January 2011

Academic Editor: Gerald A. Colvin

Copyright ( 2011 Martin H. Maurer. This is an open access article distributed under the Creative Commons Attribution License, which permits unrestricted use, distribution, and reproduction in any medium, provided the original work is properly cited.

\begin{abstract}
Mesenchymal stem cells (MSCs) are pluripotent cells isolated from the bone marrow and various other organs. They are able to proliferate and self-renew, as well as to give rise to progeny of at least the osteogenic, chondrogenic, and adipogenic lineages. Despite this functional definition, MSCs can also be defined by their expression of a distinct set of cell surface markers. In the current paper, studies investigating the proteome of human MSCs are reviewed with the aim to identify common protein markers of MSCs. The proteomic analysis of MSCs revealed a distinct set of proteins representing the basic molecular inventory, including proteins for (i) cell surface markers, (ii) the responsiveness to growth factors, (iii) the reuse of developmental signaling cascades in adult stem cells, (iv) the interaction with molecules of the extracellular matrix, (v) the expression of genes regulating transcription and translation, (vi) the control of the cell number, and (vii) the protection against cellular stress.
\end{abstract}

\section{Why Do We Study MSC Proteomes?}

The human bone marrow consists of a heterogenous group of cells, including hematopoietic stem cells (HSCs), endothelial cells, fibroblasts, adipocytes, and osteogenic cells. Additionally, in recent years, another group of cells has been found which are able to proliferate and self-renew, as well as differentiate into cells of the mesenchym such as osteoblasts, chondrocytes, and adipocytes (Figure 1). These cells were called mesenchymal stem cells (MSCs), but there is a significant controversy about a consensus definition of MSCswhich at least can be seen in the large number of names for these cells, all used simultaneously, for example, mesenchymal stem cells, mesenchymal stromal cells, marrow stromal cells, mesodermal progenitor cells, marrow-isolated adult multilineage inducible (MIAMI) cells, or CFU-F (colonyforming unit fibroblastic) (reviewed and discussed in [1]).

The interest in MSCs has risen since there is hope to use these cells in regenerative medicine, for example, in acute myocardial infarction and cardiovascular disease, diabetes, stroke, kidney disease, or immunomodulatory disease such as graft-versus-host disease (GvHD) [2-4]. Therefore, it is necessary to know the set of proteins which is responsible for the cellular functions. In recent years, proteomics emerged as a large-scale screening tool for both creating a protein inventory and to identify protein functionality. Major techniques of proteomics include two-dimensional gel electrophoresis for the separation of proteins and various mass spectrometry techniques for sequence identification [5].

In this paper, I will concentrate on the proteomic analysis of human MSCs, explicitly excluding MSCs isolated from other species (with several exceptions), or other stem cells differentiated into mesenchymal tissues in a strict sense, which are bone, cartilage, and fat, as well as MSCs differentiated into nonmesenchymal tissues such as neural or glial cells.

\section{The Dilemma of a Consensus Definition of Mesenchymal Stem Cells: Heterogenous Origins and Properties}

A functional definition of MSCs seems to be a straightforward approach, with MSCs fulfilling the following two criteria: (i) MSCs can be propagated in cell culture, where they adhere to the plastic well surface, and (ii) they can be stimulated to differentiate into osteoblasts, chondrocytes, and adipocytes. This definition is similar to the functional definition of other stem cell types, for example, for neural stem cells [6]. Of note, this definition excludes the transdifferentiation potential of MSCs into stem cells of other lineages, such as hematopoietic stem cells (HSCs), neural stem cells (NSCs), or epithelial stem cells. The concept of 


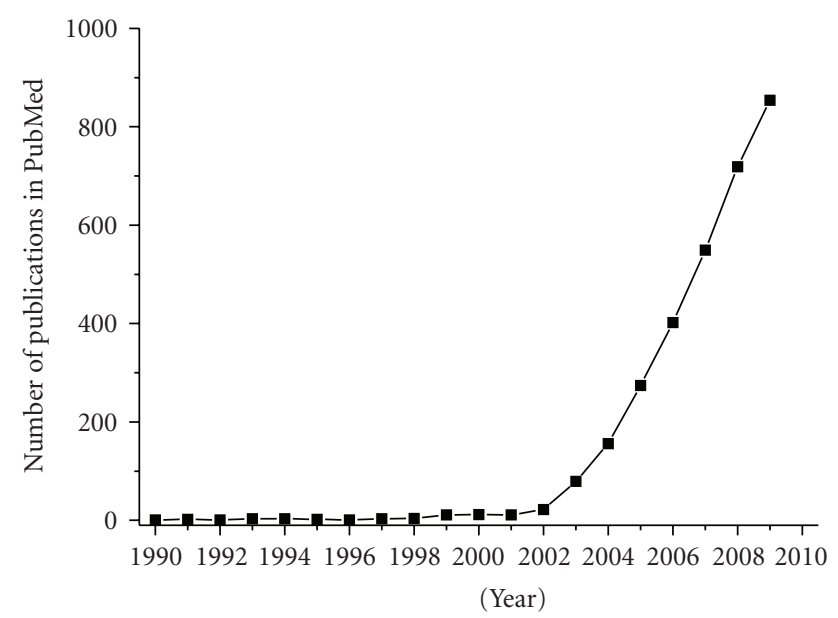

Figure 1: Scientific publications in PubMed about MSCs. The PubMed database was searched for the terms "mesenchymal stem cell," "mesenchymal stromal cell," and "MSC" for the years 19902009. The emerging interest in MSCs is seen by the increasing number of publications.

stem cell transdifferentiation is still under discussion and may be an artificial one $[1,7-10]$.

Besides the bone marrow, cells with regenerative potential have been isolated from the skeletal muscle [15], adipose tissue [16], umbilical cord [17], dental pulp [18], synovia [19], the circulatory system [20], and amniotic fluid [21] as well as fetal blood, liver, bone marrow, and lung [22-24]. The list (exceeding more than 30 organs by now) only shows that the tissue of origin cannot be used for the characterization of MSCs. Thus, we will concentrate in this paper on cells isolated from the bone marrow (BM-MSCs).

\section{Cytochemical and Immunochemical Characterization}

The cellular morphology played a major part in identifying MSCs in vitro. In general, immature MSCs appear as small, spindle-shaped cells, whereas mature MSCs display as larger cells with a flat, polygonal morphology. With regard to cellular staining techniques, MSCs were stained positive with sudan black, alkaline phosphatase, collagen IV, and fibronectin, whereas they are negative to esterase staining $[25,26]$.

With regard to immunocytochemical methods, the murine IgM monoclonal antibody STRO-1 identified bone marrow stromal cells distinct from HSCs [27]. Soon, several other surface proteins were identified which are specific for BM-MSCs [28]. In recent years, a panel of cell surface antigens emerged to characterize MSCs (compiled in [4]; see also Figure 2). According to these panels, negative and positive selection markers of MSCs have been defined. MSCs do not express CD34, CD45, CD117 (cKit), HLA class I, and HLA-DR antigens, whereas they are positive for CD13, CD29, CD44, CD73, CD90, CD105, and CD166. These markers are located in the cell membrane of the MSCs, and antibodies are readily available for FACS analysis and sorting.

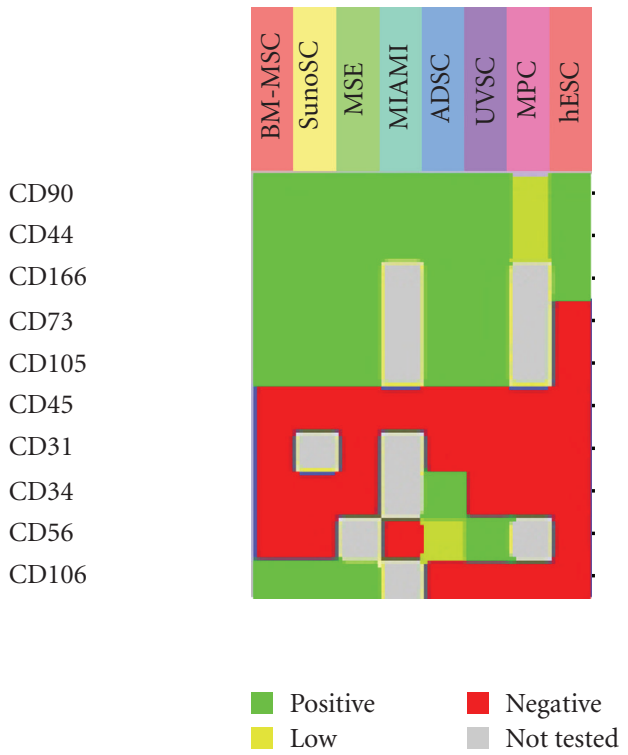

FIgure 2: Expression of cell surface markers of MSCs. Hierarchical clustering of the expression of cell surface markers of MSCs from different MSCs preparations. Cells were termed "mesenchymal stem cells (MSCs)" [11], "mesodermal progenitor cells (MPCs)" [12], "marrow-isolated adult multilineage inducible (MIAMI) cells" [13], and "bone marrow-isolated mesenchymal stem cells (BM-MSCs)," "adipose tissue-isolated stem cells (ADSCs)," "synovia-derived stem cells (SynoSCs)," "umbilical vein stem cells (UVSCs)," and "human embryonic stem cells (hESCs)" [14]. The figure shows the different cell preparations in columns and the surface antigens in rows. The green color codes for positive expression, yellow codes for low expression, red codes for no expression, and grey color stands for not determined in the respective experiments. It is seen that the cells can be defined by the presence and absence of a distinct pattern of cell surface markers, that is, CD34(-), CD45(-), CD117(-) (cKit), CD44(+), CD90(+), and CD166(+).

\section{Proteomics of Bone Marrow-Derived MSCs}

4.1. Proteome Inventories. In a recent review, the basic protein inventory of a "typical" MSC has been compiled [29], including specific proteins of the cellular metabolism, ion transport, and their respective receptors, or channels, cytokines, chemokines, growth factors, and their respective receptors, proteins of stroma-dependent hematopoiesis, extracellular matrix and connective tissue proteins, calcium homeostasis, cell cycle regulation and cellular aging, transport proteins, protein for posttranslational protein modifications, cellular detoxification, genomic transcription and translation pathways, a specific set of cell surface molecules (see also Figure 2), differentiation and development, structural components and cytoskeleton, and folding of proteins and stress response proteins (chaperones). Although no single molecule has been identified as a specific marker for MSCs, and all proteins of the list have also been found in other cell expression studies, the set of proteins and their expression arrangement is specific for MSCs.

Comparing two different approaches for MSC culture conditions, Wagner et al. [30] isolated MSCs from the bone 
marrow of healthy human donors and performed 2DE and subsequent MS to identify a set of 136 proteins. The authors compared the proteomic data to gene chip profiling data for an integrated approach and found, after interchanging media between the two groups after 8 days, that culture conditions reversed the gene and protein expression profiles. In conclusion, it is important to control and report culture conditions in details, since these experimental conditions highly influence gene and protein expression. This makes it difficult to compare experiments with different cell culture conditions [31].

A recent study compared the cellular proteomes of MSCs isolated from bone marrow (BM-MSC), adipose tissue (ADSC), synovial membrane (SynoSC), and umbilical vein wall (UVSC) [14], finding a close correlation of protein expression patterns between BM-MSCs and ADSCs. The authors used cells with the immunophenotype CD90(+), CD73(+), CD105(+), CD44(+), CD45(+) for BM-MSC, ADSC, SynoSC, and UVSC. Additionally, ADSC expressed CD34, and UVSC did not express CD106. The authors used 2DE to separate about 850 protein spots, of which 232 could be identified by MS.

The proteomic comparison of rat BM-MSCs from young and old animals [32] revealed the differential expression of Beta-actin FE-3, Caldesmon 1, Calponin-1, E-FABP (C-FABP), Galectin 3, Gamma synuclein, Heterogeneous nuclear ribonucleoprotein $\mathrm{Al}$ isoform a and $\mathrm{A} 2 / \mathrm{Bl}$ isoform A2, Huntingtin interacting protein $\mathrm{K}$, Myosin light chain, Peroxiredoxin 5, Pyruvate dehydrogenase (lipoamide) beta, and Transgelin. These proteins can be divided into the functional groups of cytoskeletal rearrangement, cellular ageing, and metabolism. Moreover, these proteins were also expressed under osteogenic differentiation.

A problem of MSC cultures is that the cells cannot be propagated over a longer period of time because of intrinsic differentiation programs. Within a few passages, the MSCs lose their proliferation potential. In a proteomic study using 2DE and LC-MS/MS, Lee at al. [33] found that the addition of $10 \mathrm{ng} / \mathrm{mL}$ basic fibroblast growth factor (bFGF) differentially regulated the expression of 15 proteins, of which actin-related protein $2 / 3$ complex subunit 2 (ARPC2), isoform 2 of glial fibrillary acidic protein (GFAP), laminA/C (LMNA), ubiquinol-cytochrome c reductase complex core protein 1 (UQCRC1), the multifunctional protein ADE2 (PAICS), F-actin-capping protein subunits alpha-1 (CAPZA1) and alpha-2 (CAPZA1), Septin-2 (SEPT2), and elongation factor 1-gamma (EEF1G) have been upregulated and Myosin regulatory light chain (MRLC2), desmoplakin (DSP), proteasome subunit alpha type 5 (PSMA5), and heat shock protein beta-1 (HSPB1) have been downregulated more than 2-fold. The functional classification of these proteins showed that these proteins mainly belonged to structural and cell morphology regulating groups, indicating that bFGF might regulate MSC differentiation and structure.

4.2. Myogenic Differentiation. Wang et al. used commercially available MSCs which are positive for CD105, CD166, CD29, and CD44, and negative for CD34, CD14, and CD45 in
MSCGM media complemented with fetal bovine serum [34]. They cultured the MSCs up to ten passages before 2DE. For myogenic differentiation, the authors stimulated the cells for four days with $10 \mathrm{ng} / \mathrm{mL}$ TGF- $\beta$ and identified about 30 proteins with differential expression, among them smooth muscle actin-alpha and gelsolin.

To induce myogenic differentiation in human MSCs, Kurpinski et al. [35] used $5 \mathrm{ng} / \mathrm{mL}$ TGF- $\beta 1$ and uniaxial mechanical stress. After $24 \mathrm{~h}$, the authors subjected the cells to $2 \mathrm{DE}$ and Q-TOF (quadrupole time of flight) MS. They identified 12 proteins which were upregulated, including eukaryotic translation elongation factor 2 (EF2), transforming growth factor-beta-induced protein ig-h3 (BGH3), calponin $3(\mathrm{CNN} 3)$, tropomyosin, actin-related protein 3 (ARP3), and smooth muscle actin-gamma. All of these proteins may be involved in myogenesis.

In a proteomic study investigating the secretome of murine BM-MSCs by LC-MS/MS, Sarojini et al. [36] identified 19 proteins, including fibronectin, pigment epitheliumderived factor (PEDF), collagen A2(I), myocilin, clusterin, protein-lysine 6-oxidase, biglycan, cathepsin L, peptidylprolyl cis-transisomerase, nucleobindin, procollagen cproteinase enhancer protein, collagen A1(I) chain, Dickkopfrelated protein-3 (Dkk-3), fibulin-2, $\beta$-2-microglobulin, CTLA-2-alpha protein, cystatin c, galectin-3, and moesin. In a cell migration assay, the secretome was applied to human fibroblast cultures. In this assay, the chemotactic migration towards to PEDF-containing secretome was increased, suggesting a function in tissue rearrangement.

4.3. Adipogenic Differentiation. The adipogenic differentiation of commercially available human MSCs was investigated by the addition of $10 \%$ FBS, $1 \mu \mathrm{M}$ dexamethasone, $0.5 \mathrm{mM}$ methyl-isobutylxanthine, $10 \mu \mathrm{g} / \mathrm{mL}$ h-insulin, and $10 \mathrm{mM}$ indomethacin to the cell culture media for 14 days [37]. The MSCs expressed CD90, but not CD34 and CD45. After 2DE and MALDI-TOF/MS, the authors identified syntaxin binding protein, oxysterol binding protein3- (OSBP-) related protein, phosphodiesterase PDE9A12, glycophorin, immunoglobulin kappa chain variable region, PPAR-gamma, and T-cell receptor $\mathrm{V}$-beta 4 as new or overexpressed protein spots.

In commercially available BM-MSCs, which are CD14(-), CD29(+), CD34(-), CD44(+), CD45(-), CD105(+), and CD166(+), Ju et al. investigated the whole cell proteome and membrane proteome by 2D-DIGE and LC-MS/MS during adipogenic differentiation [38]. Cells were incubated for 3 weeks in the LG-DMEM differentiation media containing $0.5 \mathrm{mM}$ 3-isobutyl-1-methylxantine, $1 \mathrm{mM}$ hydrocortisone, $0.1 \mathrm{mM}$ indomethacin, and 10\% FBS. The authors identified more than 700 proteins, including $33 \mathrm{CD}$ marker proteins, most of which are already known for MSCs, a large number of solute carriers, and several integrins, which are specialized cell surface molecules for cell-cell interaction and adhesion to the extracellular matrix. Moreover, more than 30 proteins were only identified in the differentiated MSCs, most of which were metabolic enzymes. 
4.4. Osteogenic Differentiation. In a one-dimensional proteomic study based on polyacrylamide gel electrophoresis (SDS-PAGE) protein separation and LC-MS/MS, Chiellini et al. [39] searched "secretome", that is, the cell culture supernatant, for secreted proteins of human multipotent adipose tissue-derived stem (hMADS) cells during adipogenesis and osteogenesis. The authors listed 73 proteins in the secretome, of which the plasminogen activator inhibitor PAI1 was found only in the osteogenic differentiation group, implicating a possible regulator between adipogenesis and osteogenesis.

Interestingly, the transplantation of MSCs into the transgenic PAI- $1^{-/-}$mouse increased graft survival [40]. The authors also analyzed the MSC cell culture supernatant after hypoxia by LC-MS/MS, finding 11 upregulated proteins, including brevican, IGF binding proteins 2, 4, and 5, myeloperoxidase, ceruloplasmin, biglycan, serine proteinase inhibitor El (PAI-1), vascular endothelial growth factor A, apelin, and superoxide dismutase 3 , and 9 downregulated proteins, including IGF-2 binding protein 3, serine hydroxymethyl transferase 1, hepatoma-derived growth factor, chemokine (CC motif) ligand 2, desmoplakin, apolipoprotein D, Kit oncogene, secreted frizzled-related sequence protein 1, and interferon zeta.

Sun et al. [41] subcultured BM-MSCs for 14 days in osteogenic media containing $10 \mathrm{mM} \beta$-glycerophosphate, $100 \mathrm{nM}$ dexamethasone, and $50 \mathrm{mg} / \mathrm{mL}$ ascorbic acid-2phosphate. They compared protein extracts by 2DE and MS to undifferentiated BM-MSCs, mapping more than 1,000 proteins on the gels. They found the upregulation of 8 proteins, including Annexin A1 and A2, Pyruvate kinase 3 (muscle), Enolase 1, Heat shock $27 \mathrm{kD}$ protein 1, Protein disulfide isomerase-related protein, Proteasome 26S ATPase subunit 5, and Cathepsin D, as well as the downregulation of 4 proteins, including T-complex protein 1, Proteasome $26 \mathrm{~S}$ ATPase subunit 2, Cadherin-2, and Chaperonin containing TCP1, subunit 3. Moreover, the authors compared serial subcultures to construct a time-resolved expression pattern for the MSC subcultures.

Using an immortalized human telomerase reverse transcriptase transduced MSC line (MSC-TERT), Foster et al. enriched protein extracts for membrane proteins and searched for differentially expressed proteins after osteoblastic differentiation by liquid chromatography (LC) and MS [42]. The authors identified 463 proteins, including MSCs phenotype CD71, CD105, CD166, CD44, Thy1, CD29, and CD63. Upon differentiation, the expression of 83 proteins increased, including alkaline phosphatase (ALP), versican core protein, and tenascin, and 21 proteins decreased more than 2-fold, including fatty acid synthase.

Kratchmarova et al. [43] investigated the effects of $50 \mathrm{ng} / \mathrm{mL}$ EGF, or $10 \mathrm{ng} / \mathrm{mL}$ PDGF for 3 days on the osteogenic differentiation of hMSC-TERT cells by LCMS/MS. Whereas EGF stimulated bone formation, PDGF did not. Proteins which were found only in the EGF-treated cells included EGFR, ErbB2, c-Cbl, DOC-2, Acid phosphatase 1, Ribonuclease inhibitor, CYLD, and KIAA2002, whereas proteins only found in the PDGF-treated group included
PDGFR alpha and beta, PI-3K (p85-alpha, p85-beta, p110alpha, p110-beta, p110-delta), Fyn, and Protocadherin 43.

4.5. MSCs and Cancer Proteomics. Whereas the self-renewal and multipotency of stem cells largely contribute to the hope of cell and tissue regeneration strategies in disease, recent data nourished the hypothesis that stem cells may also contribute to tumor generation. Their cancer-generating potential is not only seen in embryonic stem cells (ESCs), but also in MSCs, or in cells with similar potential, termed cancer-associated fibroblasts (CAFs) and circulating endothelial progenitors (CEPs) [44-46]. Therefore, the protein analysis of tumor-generating stem cells may contribute to the mechanisms of tumorigenesis.

With regard to the proteomic analysis of leukemic stromal cells, BM-MSCs from healthy donors were compared to leukemic cells using liquid phase IEF, 2D-DIGE, MALDIMS/MS, and iTRAQ methods $[48,49]$. The author found more than 900 proteins in the MSC samples by liquid phase IEF and resolved more than 5,000 protein spots on the $2 \mathrm{D}$ gels. In total, 34 proteins showed increased expression in leukemic stromal cells compared to nonleukemic MSCs, whereas 39 proteins showed decreased expression. Most of the differentially expressed proteins belonged to proteins with functions in transcription and metabolic regulation.

\section{Proteomics of Umbilical Cord-Derived MSCs (UCB-MSCs)}

MSCs can also be isolated from the umbilical cord blood by the same means as BM-MSCs [50]. Whereas the functional criteria remain comparable (adhesion to the plastic surface), cell surface markers differ largely and can be used to distinguish between BM-MSCs and UCB-MSCs.

One of the first studies creating a proteomic inventory of umbilical cord-derived mesenchymal stem cells (UCBMSCs) was from our own group [47]. We used CD29(+), CD44(+), CD73(+), CD90(+), HLA-class I(+), CD14(-), CD34(-), CD133(-), and HLA-class II $(-)$. We separated more than 2045 protein spots by 2DE and identified 205 proteins by MS. The identified proteins could be grouped into functional categories, such as metabolism, folding, cytoskeleton, transcription, signal transduction, protein degradation, detoxification, vesicle/protein transport, cell cycle regulation, apoptosis, and calcium homeostasis. In the direct comparison, the proteomes of this UCB-MSC preparation and of BM-MSCs are largely congruent, but there are still distinct differences in the protein expression pattern (Figure 3). Thus, the concept of the protein definition of MSCs evolved accordingly to the NSC proteomic definition $[6,51]$.

A major problem of $2 \mathrm{DE}$ is that hydrophobic proteins of the cell surface cannot easily be isolated in the same sample preparation as the hydrophilic proteins [5]. With regard to MSCs, Jeong et al. described the proteome preparation of hydrophobic proteins from UCB-MSCs [52]. The authors identified 35 proteins, providing additional insight into the cell surface composition of UCB-MSCs. 


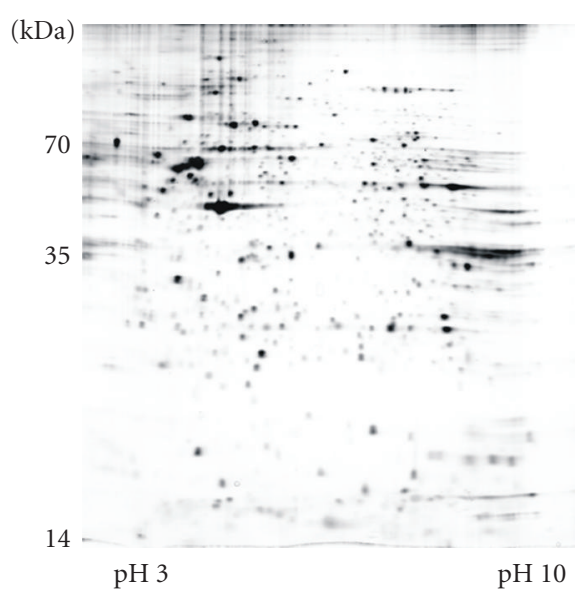

(a)

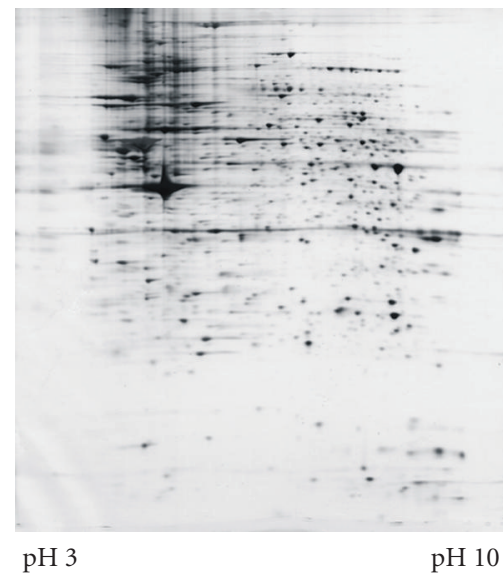

(b)

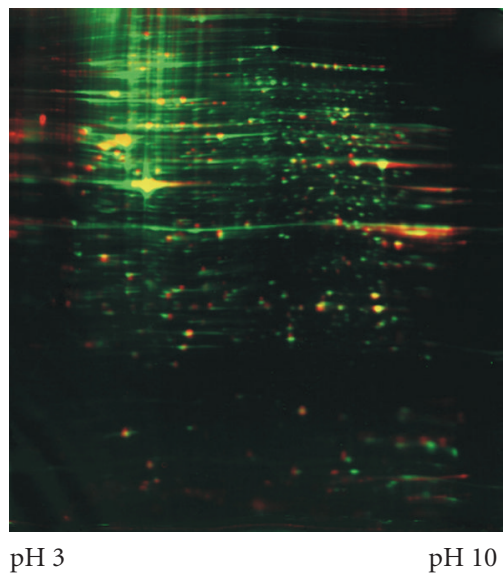

(c)

FIgure 3: Comparison of 2DE gels of BM-MSCs and UCB-MSCs. This figure shows representative 2D gel images of (a) bone marrow (BM-)MSC proteins and (b) umbilical cord blood (UCB-)MSC proteins. (c) The false-colored overlay image of the two 2D gels shows the BM-MSC proteins colored in red, and the UCB-MSC proteins colored in green. The resulting overlaid color is yellow (data from [30, 47]). The overlaid gel images show a close relationship between the two cellular proteomes, but there seem to be more proteins in the BM-MSC group as well as some differences in protein expression, as seen in the unique color in one of the gels.

A recent study compared the proteomes of UCB-MSCs, BM-MSCs, and placenta-derived MSCs by 2DE and MS [53]. The immunophenotype of all cell preparations was CD29(+), CD44(+), CD90(+), CD105(+), CD166(+), CD45(-), HLA-DR $(-), \operatorname{CD} 3(-), \operatorname{CD} 16(-), \operatorname{CD} 19(-)$, CD33(-), CD38(-), CD34(-), and CD133(-). All cell preparations could be differentiated into bone and fat cells. The authors identified six differentially regulated proteins, that is, superoxide dismutase [Mn] (MnSOD), heat shock protein HSPA9, cathepsins B and D, prohibitin, and plasminogen activator inhibitor PAI-1.

Roche et al. did not use MSCs from the umbilical cord blood, but from the umbilical cord vein walls [14]. The authors compared the proteomes to other MSC preparations (see discussion above).

\section{The Concept of Dedifferentiation Is Still a Matter of Debate}

There is one proteomic study based on 2DE and MS investigating the dedifferentiation potential of murine fibroblasts, which showed similar expression patterns with regard to cytoskeletal and cell shape remodeling, RNA export, degradation, folding, stress control, and ATP production [54]. Although dedifferentiation was initiated using the synthetic purine "reversine" (2-(4-morpholinoanilino)-6cyclohexylaminopurine), the general concept of dedifferentiation is still under discussion [55-57], also in the respective proteomic study, since no redifferentiation experiments showing the proposed multipotentiality of the dedifferentiated cells have been shown.

\section{Molecular Definitions of MSCs Using 2DE Experiments}

With regard to proteomic analyses, proteomic inventories of MSCs have been created using two-dimensional gel electrophoresis. Additionally, the events underlying cellular differentiation in vitro have been studied in osteogenesis, adipogenesis, and chondrogenesis.

Comparing the protein expression patterns, no single characteristic molecule could be identified, but the functional definition, as specified for the genomic approaches, is supported. In the light of these results, adult stem cells have all the same prerequisites, but the exact molecular composition is dependent on cell and tissue-specific factors $[51,58]$. These common prerequisites involve (Figure 4).

(i) The expression of a specific set of cell surface markers, such as CD13, CD29, CD44, CD73, CD90, CD105, and CD166, and the absence of hematopoietic stem cell markers, such as CD34, CD45, CD117 (cKit), HLA class I, and HLADR antigens.

(ii) The responsiveness to growth factors and cytokines such as PDGF, TGF- $\beta$, FGF [59], EGF, SDF-1alpha, G-CSF, GM-CSF, Angiopoietin-1, Angiopoietin-2, BMP-4, BMP-7, and IFN-gamma (reviewed in [60-62]). Of note, not all mesenchymal stem cells react to each of these molecules. A prerequisite to growth factor responsiveness is the expression of specific membrane-spanning receptors, which are able to initiate intracellular signaling cascades.

(iii) The reuse of developmental signaling cascades in adult progenitors. Nearly, 20 such pathways have been identified in adult stem cells, including Shh, Wnt, Notch/Delta, 
Reactiveness to growth factors: e.g, PDGF, TGF- $\beta$,

FGF2, EGF, SDF-1 $\alpha, \mathrm{G}(\mathrm{M})$-CSF, BMPs and Ang1/2 Cell surface markers,

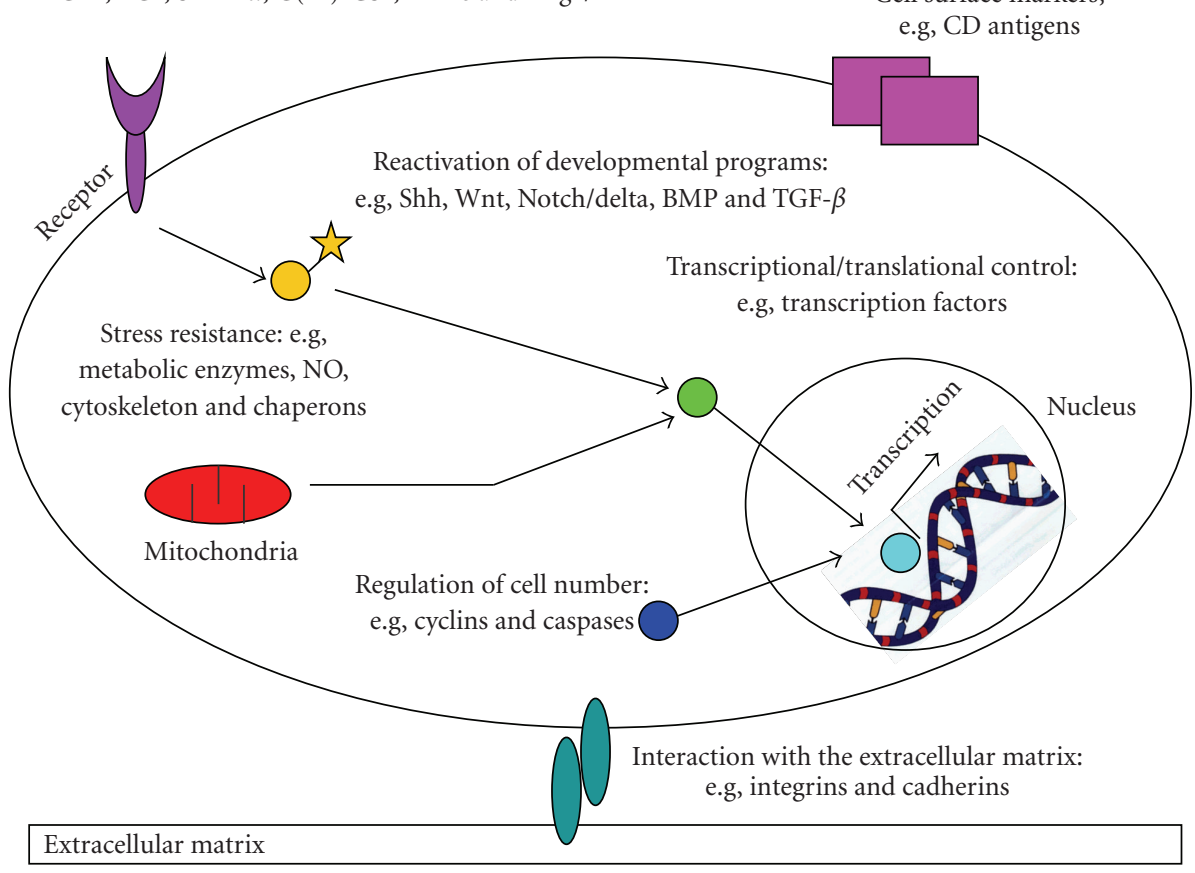

FIgure 4: Proteomic definition of MSCs-proposal for the protein inventory. The main aspects for a proteomic definition of MSCs include (i) the expression of a specific cell surface markers, (ii) the reactiveness to growth factors, (iii) the re-activation of developmental pathways, (iv) the interaction with the extracellular matrix, (v) the regulation of transcription and translation, (vi) the regulation of cell number, and (vii) the machinery for resistance to cellular stress (modified from $[6,51]$ ).

BMP, and TGF- $\beta$ (reviewed in $[60,61,63]$ ). Interestingly, the activation of these pathways in the adult may have different functions compared to the embryonic developmental pathways.

(iv) The interaction with molecules of the extracellular matrix (reviewed in $[64,65]$ ). During differentiation and maturation, stem cells migrate, extend processes, attach to the extracellular matrix, and adhere to nearby microenvironmental surfaces. Therefore, they require the expression of motor proteins, lytic enzymes, and enzymes for providing metabolic energy. Several extracellular matrix proteins mediating cell-cell interaction have been identified in mesenchymal stem cells, such as integrins and cadherins.

(v) The expression of genes regulating transcription and translation (reviewed in [66-69]). Although no specific proteins in the categories of transcription factors, DNA or RNA binding proteins, and chromatin remodeling enzymes can be named, mesenchymal stem cells need these molecules to change the cellular phenotype from its undifferentiated form to the new functional requirements of a differentiated mature cell.

(vi) Mechanisms for controlling cell number. Mainly three processes regulate stem numbers: Mitosis (proliferation), differentiation, and apoptosis (programmed cell death). An essential process is also asymmetric cell division, which regulates the stem cell pool. In this context, typical proteins regulating cell number are caspases and cyclins. (vii) The protection against cellular stress (reviewed in [70]), which involves metabolic deprivation, $\mathrm{NO}$ and $\mathrm{O}_{2}$ toxicity, DNA damage, mechanical distortion, hypo- and hyperthermia, or hypoxia. Proteins in this category have been identified in the groups of molecular chaperones, NOdetoxifying enzymes, and components of the proteasome.

Of note, although mesenchymal stem cells express these molecules, different proteins can be found in different cellular phenotypes. This heterogeneity in the protein expression pattern is supported by protein expression studies comparing mesenchymal stem cells isolated from different organs [14].

\section{Conclusions}

Human MSCs represent a heterogenous group of stem cells with a unique potential to differentiate into mesenchymal tissue. In this paper, proteomic experiments investigating human MSCs have been reviewed with the purpose to find common protein expression patterns. Six functional groups of proteins have been identified which build the prerequisite of an MSC proteomics inventory, including proteins for growth factor responsiveness, reuse of developmental pathways, regulation of transcription and translation, mechanisms controlling cell numbers, and protection against cellular stress. The composition of this set is unique, but not exclusive, for human MSCs. 


\section{Abbreviations}

2DE: Two-dimensional gel electrophoresis

ADSC: $\quad$ Adipose tissue-isolated stem cells

BM-MSC: Bone marrow-derived mesenchymal stem/stromal cell

DIGE: Differential gel electrophoresis

FACS: $\quad$ Fluorescence-activated cell sorting

hESC: Human embryonic stem cells

hMADS: Human multipotent adipose tissue-derived stem cells

HSC: $\quad$ Hematopoietic stem cell

iTRAQ: Isobaric tags for relative and absolute quantification

LC: $\quad$ Liquid chromatography

MS: $\quad$ Mass spectrometry

MS/MS: Tandem mass spectrometry

MSC: Mesenchymal stem/stromal cell

NSC: $\quad$ Neural stem cell

Q-TOF: Quadrupole time-of-flight

SDS-PAGE: Sodium dodecyl sulfate polyacrylamide gel electrophoresis

SynoSC: Synovia-derived stem cells

UCB: $\quad$ Umbilical cord blood

UVSC: Umbilical vein stem cells

CAF: $\quad$ Cancer-associated fibroblasts

CEP: Circulating endothelial progenitors.

\section{Acknowledgments}

The author was supported by grants of the European Union within the Framework Program 7, the Germany Ministry of Research and Education (BMBF) within the National Genome Research Network NGFN-2, the German Research Foundation DFG, the intramural program of the Medical Faculty of the University of Heidelberg, the Steuben-Schurz Society, and the Estate of Friedrich Fischer.

\section{References}

[1] D. J. Prockop, "Repair of tissues by adult stem/progenitor cells (MSCs): controversies, myths, and changing paradigms," Molecular Therapy, vol. 17, no. 6, pp. 939-946, 2009.

[2] National Institutes of Health, "Regenerative Medicine," 2006, http://stemcells.nih.gov/staticresources/info/scireport/PDFs/ Regenerative_Medicine_2006.pdf.

[3] M. E. Bernardo, F. Locatelli, and W. E. Fibbe, "Mesenchymal stromal cells: a novel treatment modality for tissue repair," Annals of the New York Academy of Sciences, vol. 1176, pp. 101$117,2009$.

[4] H. K. Salem and C. Thiemermann, "Mesenchymal stromal cells: current understanding and clinical status," Stem Cells, vol. 28, no. 3, pp. 585-596, 2010.

[5] M. H. Maurer and W. Kuschinsky, "Chapter 7.1: proteomics," in Handbook of Neurochemistry and Molecular Neurobiology, A. Lajtha, G. E. Gibson, and G. A. Dienel, Eds., vol. 5 of Brain Energetics. Integration of Cellular and Molecular Processes, pp. 737-769, Springer, New York, NY, USA, 2007.

[6] M. H. Maurer and W. Kuschinsky, "Screening the brain: molecular fingerprints of neural stem cells," Current Stem Cell Research \& Therapy, vol. 1, no. 1, pp. 65-77, 2006.
[7] M. Raff, "Adult stem cell plasticity: fact or artifact?" Annual Review of Cell and Developmental Biology, vol. 19, pp. 1-22, 2003.

[8] L. Jackson, D. Jones, P. Scotting, and V. Sottile, "Adult mesenchymal stem cells: differentiation potential and therapeutic applications," Journal of Postgraduate Medicine, vol. 53, no. 2, pp. 121-127, 2007.

[9] C. Krabbe, J. Zimmer, and M. Meyer, "Neural transdifferentiation of mesenchymal stem cells-a critical review," Acta Pathologica, Microbiologica et Immunologica Scandinavica, vol. 113, no. 11-12, pp. 831-844, 2005.

[10] N. H. Zech, "Plasticity of stem cells: cell-fusion versus transdifferentiation," Journal fur Reproduktionsmedizin und Endokrinologie, vol. 2, no. 4, pp. 239-245, 2005.

[11] M. F. Pittenger, A. M. Mackay, S. C. Beck et al., "Multilineage potential of adult human mesenchymal stem cells," Science, vol. 284, no. 5411, pp. 143-147, 1999.

[12] M. Reyes, T. Lund, T. Lenvik, D. Aguiar, L. Koodie, and C. M. Verfaillie, "Purification and ex vivo expansion of postnatal human marrow mesodermal progenitor cells," Blood, vol. 98, no. 9, pp. 2615-2625, 2001.

[13] G. D’Ippolito, S. Diabira, G. A. Howard, P. Menei, B. A. Roos, and P. C. Schiller, "Marrow-isolated adult multilineage inducible (MIAMI) cells, a unique population of postnatal young and old human cells with extensive expansion and differentiation potential," Journal of Cell Science, vol. 117, no. 14, pp. 2971-2981, 2004.

[14] S. Roche, B. Delorme, R. A. J. Oostendorp et al., "Comparative proteomic analysis of human mesenchymal and embryonic stem cells: towards the definition of a mesenchymal stem cell proteomic signature," Proteomics, vol. 9, no. 2, pp. 223-232, 2009.

[15] J. T. Williams, S. S. Southerland, J. Souza, A. F. Calcutt, and R. G. Cartledge, "Cells isolated from adult human skeletal muscle capable of differentiating into multiple mesodermal phenotypes," American Surgeon, vol. 65, no. 1, pp. 22-26, 1999.

[16] P. A. Zuk, M. Zhu, H. Mizuno et al., "Multilineage cells from human adipose tissue: implications for cell-based therapies," Tissue Engineering, vol. 7, no. 2, pp. 211-228, 2001.

[17] A. Erices, P. Conget, and J. J. Minguell, "Mesenchymal progenitor cells in human umbilical cord blood," British Journal of Haematology, vol. 109, no. 1, pp. 235-242, 2000.

[18] S. Gronthos, M. Mankani, J. Brahim, P. G. Robey, and S. Shi, "Postnatal human dental pulp stem cells (DPSCs) in vitro and in vivo," Proceedings of the National Academy of Sciences of the United States of America, vol. 97, no. 25, pp. 13625-13630, 2000.

[19] C. De Bari, F. Dell'Accio, P. Tylzanowski, and F. P. Luyten, "Multipotent mesenchymal stem cells from adult human synovial membrane," Arthritis and Rheumatism, vol. 44, no. 8, pp. 1928-1942, 2001.

[20] S. A. Kuznetsov, M. H. Mankani, S. Gronthos, K. Satomura, P. Bianco, and P. G. Robey, "Circulating skeletal stem cells," Journal of Cell Biology, vol. 153, no. 5, pp. 1133-1140, 2001.

[21] P. S. In 't Anker, S. A. Scherjon, C. Kleijburg-van der Keur et al., "Amniotic fluid as a novel source of mesenchymal stem cells for therapeutic transplantation," Blood, vol. 102, no. 4, pp. 1548-1549, 2003.

[22] C. G. Fan, F. W. Tang, Q. J. Zhang et al., "Characterization and neural differentiation of fetal lung mesenchymal stem cells," Cell Transplantation, vol. 14, no. 5, pp. 311-321, 2005.

[23] C. Campagnoli, I. A. G. Roberts, S. Kumar, P. R. Bennett, I. Bellantuono, and N. M. Fisk, "Identification of mesenchymal stem/progenitor cells in human first-trimester fetal blood, 
liver, and bone marrow," Blood, vol. 98, no. 8, pp. 2396-2402, 2001.

[24] W. A. Noort, A. B. Kruisselbrink, P. S. In't Anker et al., "Mesenchymal stem cells promote engraftment of human umbilical cord blood-derived CD34 $(+)$ cells in NOD/SCID mice," Experimental Hematology, vol. 30, no. 8, pp. 870-878, 2002.

[25] H. Castro-Malaspina, R. E. Gay, and G. Resnick, "Characterization of human bone marrow fibroblast colony-forming cells (CFU-F) and their progeny," Blood, vol. 56, no. 2, pp. 289-301, 1980.

[26] A. J. Friedenstein, "Stromal mechanisms of bone marrow: cloning in vitro and retransplantation in vivo," Hamatologie und Bluttransfusion, vol. 25, pp. 19-29, 1980.

[27] P. J. Simmons and B. Torok-Storb, "Identification of stromal cell precursors in human bone marrow by a novel monoclonal antibody, STRO-1," Blood, vol. 78, no. 1, pp. 55-62, 1991.

[28] W. Vogel, F. Grünebach, C. A. Messam, L. Kanz, W. Brugger, and H. J. Bühring, "Heterogeneity among human bone marrow-derived mesenchymal stem cells and neural progenitor cells," Haematologica, vol. 88, no. 2, pp. 126-133, 2003.

[29] W. P. Hye, J. S. Shin, and C. W. Kim, "Proteome of mesenchymal stem cells," Proteomics, vol. 7, no. 16, pp. 28812894, 2007.

[30] W. Wagner, R. E. Feldmann, A. Seckinger et al., "The heterogeneity of human mesenchymal stem cell preparationsevidence from simultaneous analysis of proteomes and transcriptomes," Experimental Hematology, vol. 34, no. 4, pp. 536$548,2006$.

[31] A. D. Ho, W. Wagner, and W. Franke, "Heterogeneity of mesenchymal stromal cell preparations," Cytotherapy, vol. 10, no. 4, pp. 320-330, 2008.

[32] G. Kasper, L. Mao, S. Geissler et al., "Insights into mesenchymal stem cell aging: involvement of antioxidant defense and actin cytoskeleton," Stem Cells, vol. 27, no. 6, pp. 1288-1297, 2009.

[33] S. K. Lee, Y. Kim, S. S. Kim et al., "Differential expression of cell surface proteins in human bone marrow mesenchymal stem cells cultured with or without basic fibroblast growth factor containing medium," Proteomics, vol. 9, no. 18, pp. 4389-4405, 2009.

[34] D. Wang, J. S. Park, J. S. F. Chu et al., "Proteomic profiling of bone marrow mesenchymal stem cells upon transforming growth factor $\beta 1$ stimulation," Journal of Biological Chemistry, vol. 279, no. 42, pp. 43725-43734, 2004.

[35] K. Kurpinski, J. Chu, D. Wang, and S. Li, "Proteomic profiling of mesenchymal stem cell responses to mechanical strain and TGF- $\beta 1$," Cellular and Molecular Bioengineering, vol. 2, no. 4, pp. 606-614, 2009.

[36] H. Sarojini, R. Estrada, H. Lu et al., "PEDF from mouse mesenchymal stem cell secretome attracts fibroblasts," Journal of Cellular Biochemistry, vol. 104, no. 5, pp. 1793-1802, 2008.

[37] H. K. Lee, B. H. Lee, S. A. Park, and C. W. Kim, "The proteomic analysis of an adipocyte differentiated from human mesenchymal stem cells using two-dimensional gel electrophoresis," Proteomics, vol. 6, no. 4, pp. 1223-1229, 2006.

[38] AH. J. Ju, K. M. Ko, S. P. Hyung et al., "Membrane proteomic analysis of human mesenchymal stromal cells during adipogenesis," Proteomics, vol. 7, no. 22, pp. 4181-4191, 2007.

[39] C. Chiellini, O. Cochet, L. Negroni et al., "Characterization of human mesenchymal stem cell secretome at early steps of adipocyte and osteoblast differentiation," BMC Molecular Biology, vol. 9, article 26, 2008.
[40] I. B. Copland, S. Lord-Dufour, J. Cuerquis et al., "Improved autograft survival of mesenchymal stromal cells by plasminogen activator inhibitor 1 inhibition," Stem Cells, vol. 27, no. 2, pp. 467-477, 2009.

[41] H. J. Sun, Y. Y. Bahk, Y. R. Choi, J. H. Shim, S. H. Han, and J. W. Lee, "A proteomic analysis during serial subculture and osteogenic differentiation of human mesenchymal stem cell," Journal of Orthopaedic Research, vol. 24, no. 11, pp. 2059-2071, 2006.

[42] L. J. Foster, P. A. Zeemann, C. Li, M. Mann, O. N. Jensen, and M. Kassem, "Differential expression profiling of membrane proteins by quantitative proteomics in a human mesenchymal stem cell line undergoing osteoblast differentiation," Stem Cells, vol. 23, no. 9, pp. 1367-1377, 2005.

[43] I. Kratchmarova, B. Blagoev, M. Haack-Sorensen, M. Kassem, and M. Mann, "Cell Signalling: mechanism of divergent growth factor effects in mesenchymal stem cell differentiation," Science, vol. 308, no. 5727, pp. 1472-1477, 2005.

[44] P. J. Mishra, P. J. Mishra, J. W. Glod, and D. Banerjee, "Mesenchymal stem cells: flip side of the coin," Cancer Research, vol. 69, no. 4, pp. 1255-1258, 2009.

[45] M. Battiwalla and P. Hematti, "Mesenchymal stem cells in hematopoietic stem cell transplantation," Cytotherapy, vol. 11, no. 5, pp. 503-515, 2009.

[46] E. H. Lin, Y. Jiang, Y. Deng, R. Lapsiwala, T. Lin, and C. A. Blau, "Cancer stem cells, endothelial progenitors, and mesenchymal stem cells: "seed and soil" theory revisited," Gastrointestinal Cancer Research, vol. 2, no. 4, pp. 169-174, 2008.

[47] R. E. Feldmann, K. Bieback, M. H. Maurer et al., "Stem cell proteomes: a profile of human mesenchymal stem cells derived from umbilical cord blood," Electrophoresis, vol. 26, no. 14, pp. 2749-2758, 2005.

[48] B. Seshi, "An integrated approach to mapping the proteome of the human bone marrow stromal cell," Proteomics, vol. 6, no. 19, pp. 5169-5182, 2006.

[49] B. Seshi, "Proteomics strategy based on liquid-phase IEF and 2-D DIGE: application to bone marrow mesenchymal progenitor cells," Proteomics, vol. 7, no. 12, pp. 1984-1999, 2007.

[50] K. Bieback, S. Kern, H. Klüter, and H. Eichler, "Critical parameters for the isolation of mesenchymal stem cells from umbilical cord blood," Stem Cells, vol. 22, no. 4, pp. 625-634, 2004.

[51] M. H. Maurer, "Neural stem cells: a functional definition based on proteomics," Electronic Journal of Biology, vol. 4, no. 2, pp. 43-46, 2008.

[52] JU. A. Jeong, Y. Lee, W. Lee et al., "Proteomic analysis of the hydrophobic fraction of mesenchymal stem cells derived from human umbilical cord blood," Molecules and Cells, vol. 22, no. 1, pp. 36-43, 2006.

[53] G. Li, X. A. Zhang, H. Wang et al., "Comparative proteomic analysis of mesenchymal stem cells derived from human bone marrow, umbilical cord, and placenta: implication in the migration," Proteomics, vol. 9, no. 1, pp. 20-30, 2009.

[54] C. Fania, L. Anastasia, M. Vasso et al., "Proteomic signature of reversine-treated murine fibroblasts by 2-D difference gel electrophoresis and MS: possible associations with cell signalling networks," Electrophoresis, vol. 30, no. 12, pp. 21932206, 2009.

[55] I. L. Weissman, "Stem cells: units of development, units of regeneration, and units in evolution," Cell, vol. 100, no. 1, pp. 157-168, 2000. 
[56] A. J. Wagers and I. L. Weissman, "Plasticity of adult stem cells," Cell, vol. 116, no. 5, pp. 639-648, 2004.

[57] E. L. Herzog, LI. Chai, and D. S. Krause, "Plasticity of marrowderived stem cells," Blood, vol. 102, no. 10, pp. 3483-3493, 2003.

[58] D. E. Discher, D. J. Mooney, and P. W. Zandstra, "Growth factors, matrices, and forces combine and control stem cells," Science, vol. 324, no. 5935, pp. 1673-1677, 2009.

[59] F. Ng, S. Boucher, S. Koh et al., "PDGF, tgf-. And FGF signaling is important for differentiation and growth of mesenchymal stem cells (mscs): transcriptional profiling can identify markers and signaling pathways important in differentiation of MSCs into adipogenic, chondrogenic, and osteogenic lineages," Blood, vol. 112, no. 2, pp. 295-307, 2008.

[60] F. Deschaseaux, L. Sensébé, and D. Heymann, "Mechanisms of bone repair and regeneration," Trends in Molecular Medicine, vol. 15, no. 9, pp. 417-429, 2009.

[61] W. Han, Y. Yu, and Y. L. Xin, "Local signals in stem cell-based bone marrow regeneration," Cell Research, vol. 16, no. 2, pp. 189-195, 2006.

[62] M. H. Maurer, W. R. Schäbitz, and A. Schneider, "Old friends in new constellations - the hematopoetic growth factors GCSF, GM-CSF, and EPO for the treatment of neurological diseases," Current Medicinal Chemistry, vol. 15, no. 14, pp. 1407-1411, 2008.

[63] J. E. Dennis and P. Charbord, "Origin and differentiation of human and murine stroma," Stem Cells, vol. 20, no. 3, pp. 205214, 2002.

[64] S. M. Richardson, J. A. Hoyland, R. Mobasheri, C. Csaki, M. Shakibaei, and A. Mobasheri, "Mesenchymal stem cells in regenerative medicine: opportunities and challenges for articular cartilage and intervertebral disc tissue engineering," Journal of Cellular Physiology, vol. 222, no. 1, pp. 23-32, 2010.

[65] S. Fruehauf, K. Srbic, R. Seggewiss, J. Topaly, and A. D. Ho, "Functional characterization of podia formation in normal and malignant hematopoietic cells," Journal of Leukocyte Biology, vol. 71, no. 3, pp. 425-432, 2002.

[66] S. R. Farmer, "Transcriptional control of adipocyte formation," Cell Metabolism, vol. 4, no. 4, pp. 263-273, 2006.

[67] D. Benayahu, U. D. Akavia, and I. Shur, "Differentiation of bone marrow stroma-derived mesenchymal cells," Current Medicinal Chemistry, vol. 14, no. 2, pp. 173-179, 2007.

[68] D. Benayahu, G. Shefer, and I. Shur, "Insights into the transcriptional and chromatin regulation of mesenchymal stem cells in musculo-skeletal tissues," Annals of Anatomy, vol. 191, no. 1, pp. 2-12, 2009.

[69] C. S. Soltanoff, S. Yang, W. Chen, and YI. P. Li, "Signaling networks that control the lineage commitment and differentiation of bone cells," Critical Reviews in Eukaryotic Gene Expression, vol. 19, no. 1, pp. 1-46, 2009.

[70] K. Ksiazek, "A comprehensive review on mesenchymal stem cell growth and senescence," Rejuvenation Research, vol. 12, no. 2, pp. 105-116, 2009. 

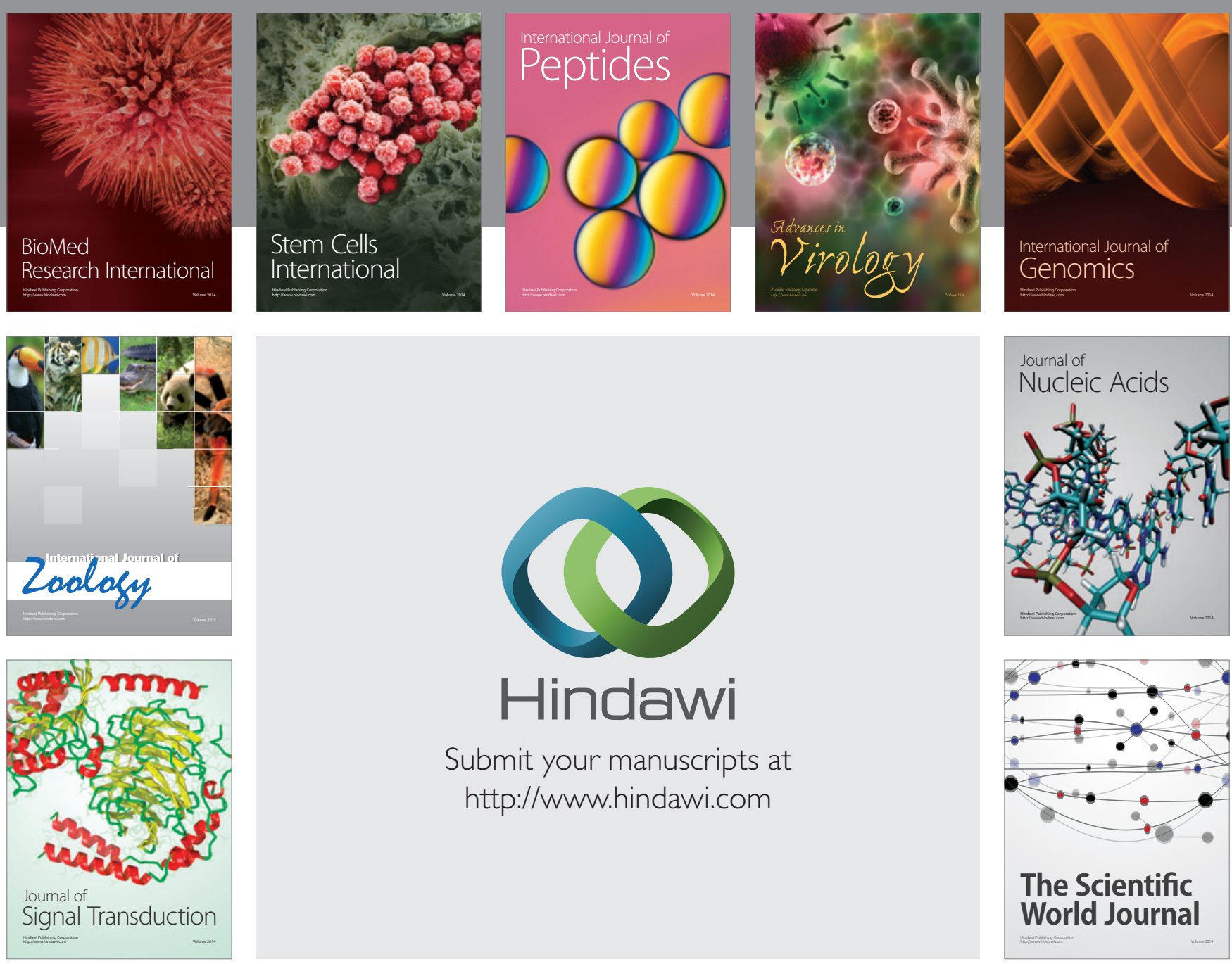

Submit your manuscripts at

http://www.hindawi.com
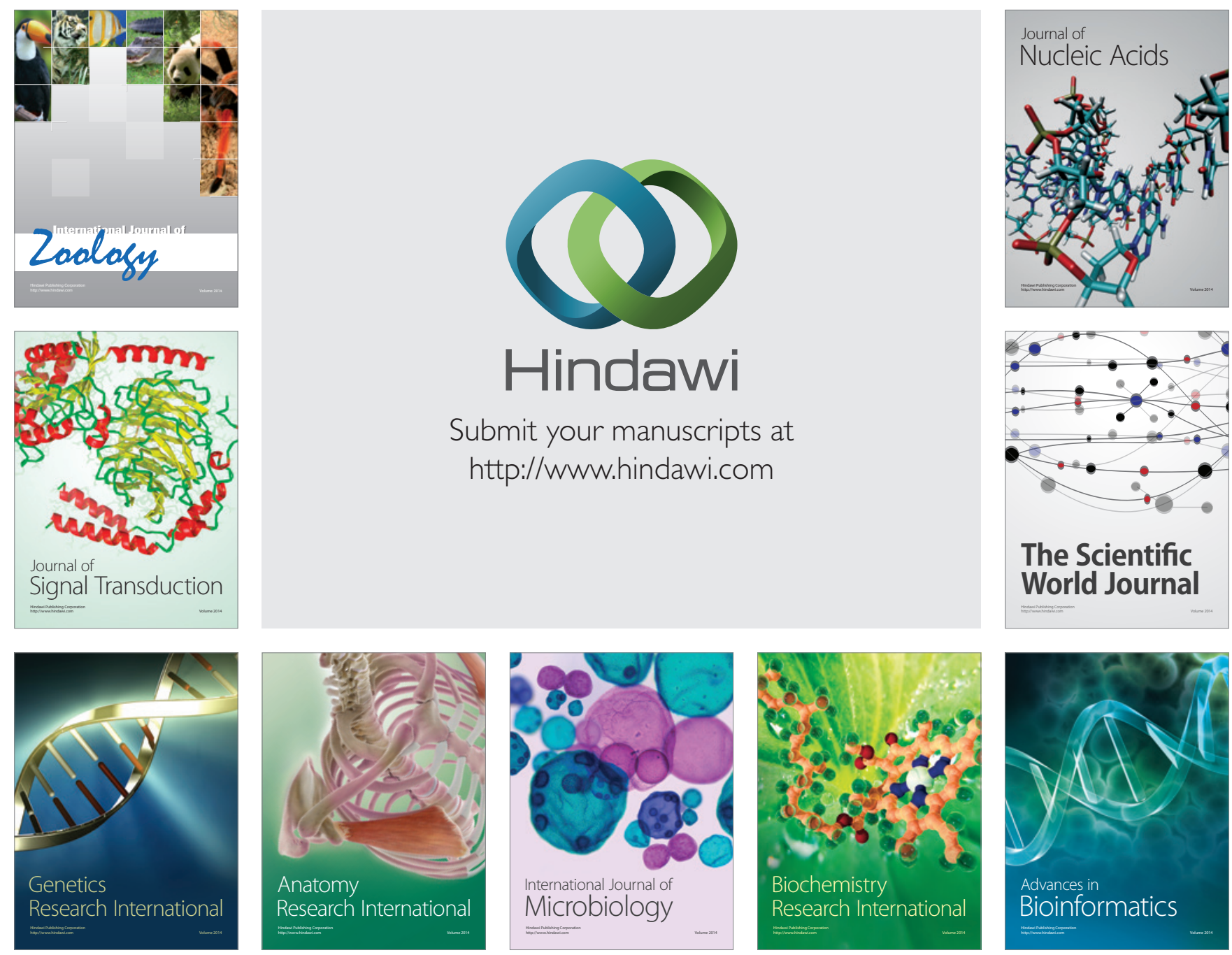

The Scientific World Journal
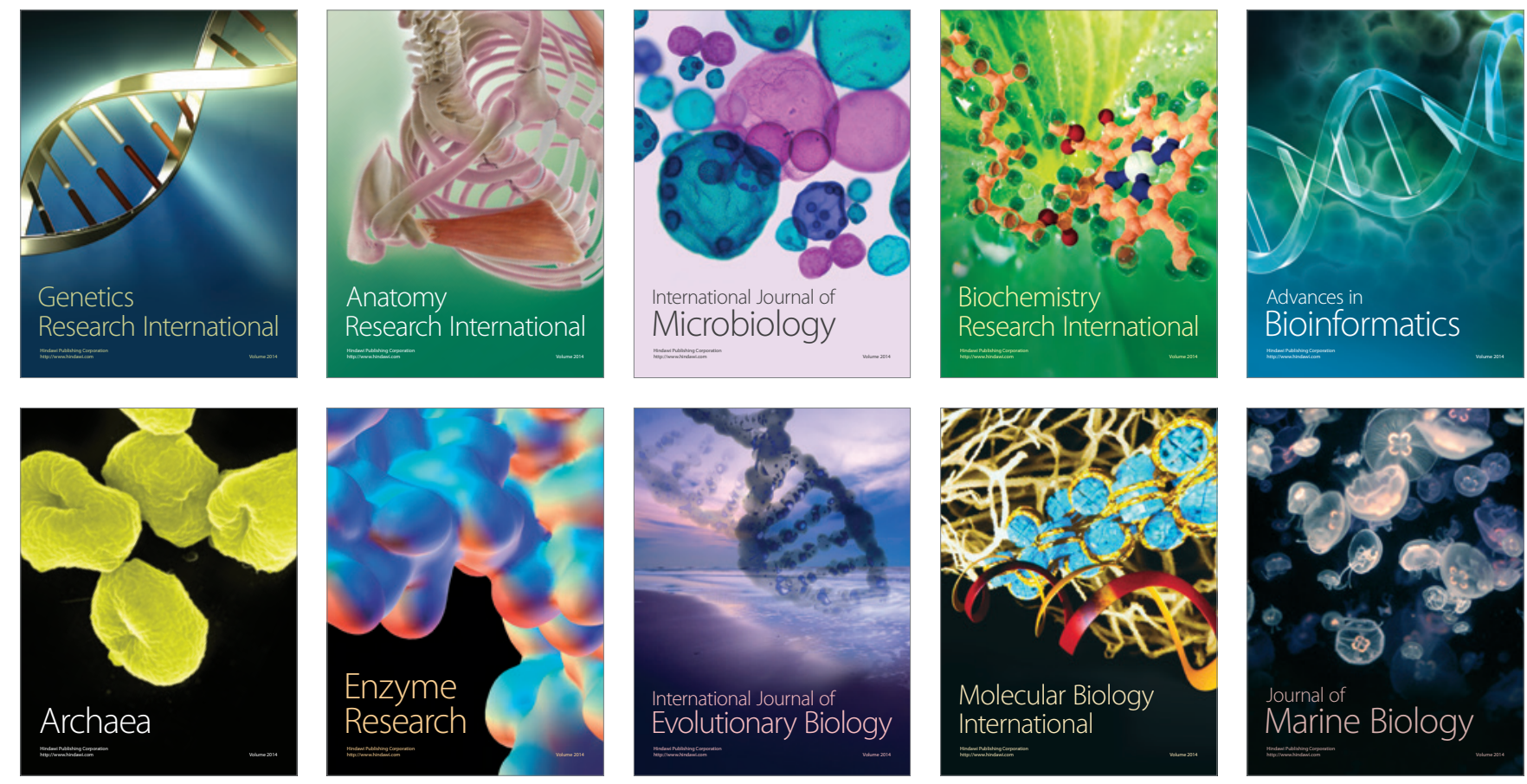